\title{
Nasopharyngeal gene expression, a novel approach to study the course of respiratory syncytial virus infection
}

\author{
Corné H. van den Kieboom ${ }^{1,2}$, Inge M.L. Ahout ${ }^{1,2}$, Aldert Zomer ${ }^{1,2}$, Kim H. Brand ${ }^{1}$, \\ Ronald de Groot ${ }^{1,2}$, Gerben Ferwerda ${ }^{1,2}$ and Marien I. de Jonge ${ }^{1,2}$
}

Affiliations: 'Laboratory of Pediatric Infectious Diseases, Dept of Pediatrics, Radboud University Medical Center, Nijmegen, The Netherlands. ${ }^{2}$ Laboratory of Medical Immunology, Dept of Laboratory Medicine, Radboud University Medical Center, Nijmegen, The Netherlands.

Correspondence: Corné van den Kieboom, Laboratory of Pediatric Infectious Diseases, Dept of Pediatrics, Radboud University Medical Center, PO Box 9101, Nijmegen 6500HB, The Netherlands.

E-mail: Corne.vandenKieboomaradboudumc.nl

ABSTRACT Respiratory syncytial virus (RSV) causes mild infections in the vast majority of children. However, in some cases, it causes severe disease, such as bronchiolitis and pneumonia. Development of severe RSV infection is determined by the host response. Therefore, the main aim of this study was to identify biomarkers associated with severe RSV infection.

To identify biomarkers, nasopharyngeal gene expression was profiled by microarray studies, resulting in the selection of five genes: ubiquitin $D$, tetraspanin 8 , mucin $13, \beta$-microseminoprotein and chemokine ligand 7.

These genes were validated by real-time quantitative PCR in an independent validation cohort, which confirmed significant differences in gene expression between mildly and severely infected and between recovery and acute patients.

Nasopharyngeal aspirate samples are regularly taken when a viral respiratory tract infection is suspected. In this article, we describe a method to discriminate between mild and severe RSV infection based on differential host gene expression. The combination of pathogen detection and host gene expression analysis in nasopharyngeal aspirates will significantly improve the diagnosis and prognosis of respiratory tract infections.

@ERSpublications

Host gene expression analysis of nasopharyngeal aspirate samples can be used to predict RSV disease severity http://ow.ly/AMxpt

This article has supplementary material available from erj.ersjournals.com

Received: May 092014 | Accepted after revision: Aug 182014 | First published online: Sept 262014

Support statement: This work was supported by Agentschap NL, module IV grant PNU10A23, the Virgo consortium (grant FES0908), The Netherlands Genomics Initiative (050-060-452) and the Technology Foundation STW (FES0901 and FES HTSM). The sponsors had no role in study design, data collection, data analysis, data interpretation, decision to publish or preparation of the manuscript.

Conflict of interest: None declared.

Copyright OERS 2015 


\section{Introduction}

Respiratory syncytial virus (RSV) is the most common cause of viral respiratory tract infections among hospitalised children. Symptoms range from a common cold to severely compromised respiratory function, causing $6-15 \%$ of the admitted children to require intensive care $[1,2]$. It is difficult to predict the course of disease in infants, especially in children under the age of 3 months. Current clinical prediction rules are based on demographic criteria and clinical symptoms [3-10] and although they may improve clinical judgement $[7,8]$, they are not routinely used in daily practice. More objective and reproducible predictions may be achieved by using biomarkers [11-15].

Recent publications showed that gene expression profiles in peripheral blood mononuclear cells represent the subject's health. Ramilo et al. [16] were able to discriminate influenza A, Escherichia coli and Streptococcus pneumoniae infection with 95\% accuracy, and distinguished E. coli from Staphylococcus aureus infection with $85 \%$ accuracy by analysing mRNA expression level in young patients. In the case of RSV infections, MeJIAS et al. [17] showed, based on blood RNA profiles of infants, a classification of infants with RSV lower respiratory tract infection (LRTI) versus rhinovirus or influenza LRTI with 95\% accuracy. Additional studies confirmed these observations and described the discovery of several biomarkers in blood by which different states of disease could be distinguished [18].

These findings may be of great value for clinical decision making. However, the studies, as described, were performed using blood samples, of which only limited volumes can be obtained from paediatric patients. Thus, it would be worthwhile to study whether other clinical materials could be used that reflect the host response and may harbour prognostic value. The nasopharynx is the point of entry of all respiratory viruses; moreover, a sample from this site is already taken to diagnose viral infections using real-time quantitative PCR (RT-qPCR) [19]. The use of nasopharyngeal aspirates (NPAs) for the measurement of host biomarker expression for prognostic purposes would be worthwhile to assess, especially as clinical laboratories would only need minimal adjustment to integrate this test into current practice. Knowledge with regards gene expression in the nasopharynx during RSV infections is limited and has been gained solely from animal models [20].

In this study, we assessed the possibility of measuring host gene expression in NPA taken from children with laboratory-confirmed RSV infections and aimed to identify markers with potential prognostic value for the course of disease.

\section{Materials and methods}

Ethics

The study was approved by the Committee on Research Involving Human Subjects of the Radboud University Medical Center (Nijmegen, the Netherlands). All clinical samples collected from patients in our cohorts were enrolled following informed consent from parents or guardians. The clinical information available to subjects being recruited for the study included a clear description of the risks and benefits of participation, indicating the required insurance and procedures, safe handling and protection of all data. All subjects were informed that there was no obligation to participate and that declining to participate or leaving the study had no adverse consequences.

\section{Study design}

Children $<5$ years of age with symptoms of viral LRTI were included. These symptoms included increased respiratory effort (e.g. tachypnoea and/or use of accessory respiratory muscles) and/or expiratory wheezing and/or crackles and/or apnoea. Retrospectively, the LRTI was confirmed by RT-qPCR on nasopharyngeal washes as described previously [19]. Patients with congenital or acquired immune deficiency, immunosuppressive medication (including $>24 \mathrm{~h}$ of glucocorticosteroids) or severe psychomotor retardation were excluded.

All subjects were recruited at two hospitals in Nijmegen. Subjects admitted were screened for symptoms typical for viral respiratory tract infection, as described previously. When RSV infection was suspected, a NPA was collected within $24 \mathrm{~h}$ of admission (acute) and parents of hospitalised children were asked permission to draw a second NPA sample 4-6 weeks after admission (recovery). Medical history, demographics and clinical parameters were collected from questionnaires and medical records. Patients were classified into three different groups based on the degree of supportive care they needed during the course of disease: no supportive care; supplemental oxygen (when oxygen saturation was $<93 \%$ for $>10 \mathrm{~min}$ ); or mechanical ventilation.

Microarrays were performed on samples from the cohort published by BRAND et al. [18] ( $\mathrm{n}=30,2006-2009)$ and subsequently selected markers were validated in the cohort of I.M.L. Ahout and co-workers (personal communication; manuscript in preparation) $(\mathrm{n}=44,2010-2012)$. 


\section{Sample collection}

After informed consent, a NPA sample was collected by introducing a catheter, connected to a collection tube and an aspiration system, into the nasopharyngeal cavity. Then, saline $(0.5-1.5 \mathrm{~mL})$ was instilled into the catheter and, while slowly retracting the catheter, the nasopharyngeal fluid was aspirated in a collection tube. Afterwards, the catheter was flushed with saline and added to the collection fluid.

The samples were kept on ice and immediately transferred to the laboratory, where they were centrifuged $(500 \times g)$ for $10 \mathrm{~min}$ at $4^{\circ} \mathrm{C}$ to spin down the mucus and cells, after which the pellet was mixed with Trizol (Life Technologies, Carlsbad, CA, USA) and stored at $-80^{\circ} \mathrm{C}$. RT-qPCR was performed using the supernatant to confirm viral aetiology of the disease [19].

\section{Microarray}

RNA was extracted from NPA in Trizol according to the manufacturer's protocol. The final RNA pellet was washed twice with $1 \mathrm{~mL}$ of $75 \%$ ethanol, air dried and resuspended in $100 \mu \mathrm{L}$ of RNAse/DNase free water. Subsequently, a clean-up of the total RNA was performed with the RNeasy Minikit (Qiagen, Hilden, Germany) according to the manufacturer's instructions. RNA integrity and quality for the microarray was assessed using a RNA 6000 Nano LabChip on an Bioanalyzer 2100 system (Agilent, Santa Clara, CA, USA).

RNA processing, target labelling and hybridisation to gene expression arrays was performed. Biotin-labelled cRNA was obtained using the One-Cycle Eukaryotic Target Labeling Assay (Affymetrix Inc., Santa Clara, CA). $15 \mu \mathrm{g}$ of fragmented, biotin-labelled cRNA was hybridised to Affymetrix GeneChip Human Genome U133 Plus 2.0 arrays according to standard Affymetrix protocol.

\section{Microarray data analysis}

Quality control analyses were performed as previously described [21]. Scanned images were inspected for artefacts, percentage of present calls $(<25 \%)$ and controls of RNA degradation. Background signal was removed using robust multichip analysis and probe intensity levels were quantile-normalised across arrays. Microarray analysis was performed using ArrayStar4 (DNAStar, Madison, WI, USA).

After the microarray data were obtained, comparisons were made based on supportive care: the need for supportive care (comparison 1) and mechanical ventilation (comparison 2). p-values were corrected for false discovery rate and, subsequently, the significant genes with a four-fold change were selected, while probes directed at noncoding sequences were excluded [22].

The selected genes were not normally distributed according to the d'Agostino and Pearson omnibus normality test $(\alpha=0.05)$ (Prism 5; Graphpad Software, La Jolla, CA, USA). Therefore, a permutation test using MultiExperiment Viewer (tm4group; Dana-Farber Cancer Institute, Boston, MA, USA) with random group samples $\left(10^{5}\right)$ was performed and the median fold change cut-off was set at $2.50 \log$. The overlapping genes between the two comparisons and the only upregulated gene were selected for validation by $\mathrm{RT}-\mathrm{qPCR}$.

\section{Gene validation by $R T-q P C R$}

Samples were defrosted on ice and RNA was extracted from NPA in Trizol according to the manufacturer's protocol. The RNA pellet was washed twice with $1 \mathrm{~mL}$ of $75 \%$ ethanol, air dried, re-suspended in $100 \mu \mathrm{L}$ of diethylpyrocarbonate-treated water. Subsequently, a clean-up was performed with the RNeasy Minikit according to the manufacturer's instructions. The elutate was treated with TURBO DNA-free (Life Technologies); subsequently, cDNA synthesised by iScript (Biorad, Hercules, CA, USA) and stored at $-20^{\circ} \mathrm{C}$.

The RT-qPCR was conducted on a CFX96 (Biorad) using Taqman gene expression assays (Hs.00197374_m1, Hs.00610327_m1, Hs.00217239_m1, Hs.00738231_m1, Hs.00171147_m1 and Hs.02758991_g1; Life Technologies) and IQ Powermix (Biorad). The PCR programme consisted of an initial $5 \mathrm{~min}$ at $96^{\circ} \mathrm{C}$, followed by 40 cycles of $15 \mathrm{~s}$ at $96^{\circ} \mathrm{C}$ and $45 \mathrm{~s}$ at $60^{\circ} \mathrm{C}$. The data generated were analysed using CFX Manager 3.0 (Biorad) and the cut-off value was set by using the single threshold mode for quantification cycle $\left(\mathrm{C}_{\mathrm{q}}\right)$ determination. The data were normalised by subtracting the $\mathrm{C}_{\mathrm{q}}$ value of the housekeeping gene, i.e. glyceraldehyde 3-phosphate dehydrogenase (GAPDH) (Hs.02758991_g1; Life Technologies).

\section{Statistical analysis}

Significance of the microarray analysis was calculated by a permutation test, with $10^{5}$ random group samples using TMEV (tm4group). Significance of the RT-qPCR-measured gene expression was calculated by Mann-Whitney U-tests using Prism 5, where a two-sided value of $\mathrm{p}<0.05$ was considered statistically significant. 
TABLE 1 Microarray cohort patient characteristics

\begin{tabular}{|c|c|c|c|c|c|}
\hline & \multirow{2}{*}{$\begin{array}{c}\text { No supportive } \\
\text { care }\end{array}$} & \multirow{2}{*}{$\begin{array}{l}\text { Supplemental } \\
\text { oxygen }\end{array}$} & \multirow{2}{*}{$\begin{array}{l}\text { Mechanical } \\
\text { ventilation }\end{array}$} & \multicolumn{2}{|c|}{ p-value } \\
\hline & & & & $\begin{array}{c}\text { Kruskal-Wallis } \\
\text { test }\end{array}$ & $\begin{array}{c}\text { Mann-Whitney } \\
\text { U-test }\end{array}$ \\
\hline Patients $\mathrm{n}$ & 10 & 10 & 10 & & \\
\hline Age days & 227 (57-279) & $135(46-337)$ & $64(41-161)$ & NS & NA \\
\hline Males n (\%) & $8(80)$ & $8(80)$ & $8(80)$ & NS & NA \\
\hline Duration of symptoms days & $7(4-9)$ & $5(3-7)$ & $5(4-5)$ & NS & NA \\
\hline Duration of oxygen therapy days & 0 & $3(1-6)$ & $9(5-11)$ & 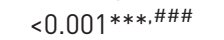 & 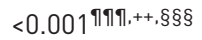 \\
\hline Length of hospital stay days & $0(0-3)$ & $5(2-7)$ & $13(7-16)$ & 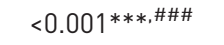 & 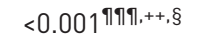 \\
\hline \multicolumn{6}{|c|}{ 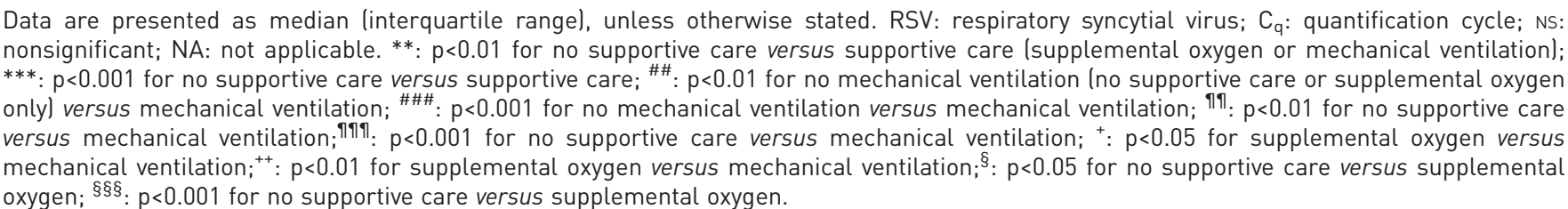 } \\
\hline
\end{tabular}

Correlation was assessed by Spearman analysis using SPSS (IBM, Armonk, NY, USA), where a two-sided value of $\mathrm{p}<0.05$ was considered statistically significant. For the patient characteristics, the Kruskal-Wallis analysis was performed; in the case of significance $(\mathrm{p}<0.05)$, we subsequently performed Mann-Whitney U-tests for individual comparisons using SPSS.

\section{Results \\ Microarray data analysis}

In this study, the clinical value of nasopharyngeal gene expression was assessed by conducting microarrays on NPAs of 30 infants with a confirmed RSV infection, both mono- (RSV) and co-infections. The patient characteristics are displayed in table 1 . No significant differences were found, except for the parameters on which the clinical categorisation was based and gestational age.

To select potential biomarkers, comparisons were made using microarray analysis with regards to the need for supportive care: supplemental oxygen (comparison 1) and the need for mechanical ventilation (comparison 2). The permutation test led to an initial selection of 53 targets (table S1) for comparison 1 and 93 targets for comparison 2 (table S2). By the subsequent introduction of a median fold change cut-off of $2.5 \mathrm{log}, 16$ genes were selected that were significantly differentially expressed in comparison 1 (table S3) and 28 genes were selected in comparison 2 (table S4). The two selections were compared and four overlapping genes were found (ubiquitin D (UBD), tetraspanin 8 (TSPAN8), mucin 13 (MUC13) and $\beta$-microseminoprotein $(M S P)$ ), which all showed downregulation. In addition to these genes, chemokine ligand 7 (CCL7) was selected as the only upregulated differentially expressed gene in the whole microarray analysis (table 2).

TABLE 2 Microarray cohort based gene selection

\begin{tabular}{|c|c|c|c|c|c|c|}
\hline Gene & Gene product & \multicolumn{2}{|c|}{ Supportive care } & \multicolumn{2}{|c|}{ Mechanical ventilation } & TaqMan gene expression assay \\
\hline UBD & Ubiquitin D & 0.00271 & -3.50 & 0.00004 & -3.54 & Hs.00197374_m1 \\
\hline TSPAN8 & Tetraspanin 8 & 0.00429 & -3.39 & 0.00157 & -2.81 & Hs.00610327_m1 \\
\hline MUC13 & Mucin 13 & 0.00593 & -3.54 & 0.00002 & -3.41 & Hs.00217239_m1 \\
\hline$M S P^{\#}$ & $\beta$-microseminoprotein & 0.00168 & -4.09 & 0.00009 & -2.83 & Hs.00738231_m1 \\
\hline CCL7 & Chemokine ligand 7 & NA & NA & 0.00061 & 2.70 & Hs.00171147_m1 \\
\hline
\end{tabular}

NA: not applicable. ${ }^{\#}$ : multiple probes were found; the average $p$-value and median fold change are displayed. 
TABLE 3 Validation cohort patient characteristics

\begin{tabular}{|c|c|c|c|c|c|}
\hline & \multirow{2}{*}{$\begin{array}{c}\text { No supportive } \\
\text { care }\end{array}$} & \multirow{2}{*}{$\begin{array}{c}\text { Supplemental } \\
\text { oxygen }\end{array}$} & \multirow{2}{*}{$\begin{array}{l}\text { Mechanical } \\
\text { ventilation }\end{array}$} & \multicolumn{2}{|c|}{ p-value } \\
\hline & & & & $\begin{array}{c}\text { Kruskal-Wallis } \\
\text { test }\end{array}$ & $\begin{array}{c}\text { Mann-Whitney } \\
\text { U-test }\end{array}$ \\
\hline Patients $\mathrm{n}$ & 7 & 21 & 14 & & \\
\hline Age days & $153(102-325)$ & $74(55-179)$ & $42(28-76)$ & $<0.05^{*}, \#$ & $<0.01^{++}$ \\
\hline Males & $5(71)$ & $10(48)$ & $7(50)$ & NS & NA \\
\hline RSV serotype & $6(86)$ & $11(52)$ & $14(100)$ & $<0.01^{\# \#}$ & $<0.05^{\S}$ \\
\hline Duration of symptoms days & $5(4-5)$ & $4(3-5)$ & $3(2-5)$ & NS & NA \\
\hline Duration of supplemental oxygen days & 0 & $3(2-4)$ & $9(7-11)$ & $<0.001^{* * *}$,\#\#\# & 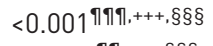 \\
\hline Length of hospital stay days & $2(0-6)$ & $5(4-8)$ & $11(10-13)$ & $<0.001 * * *$,\#\#\# & $<0.001^{1 \uparrow 1,+++, \S \S \S ~}$ \\
\hline \multicolumn{6}{|c|}{ 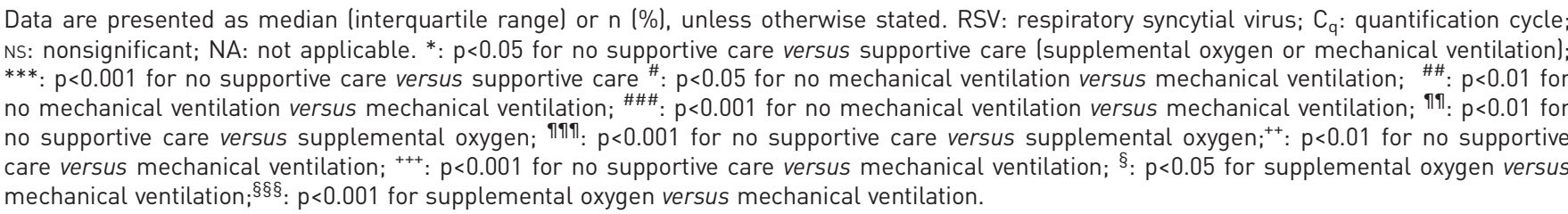 } \\
\hline
\end{tabular}

\section{Gene expression validation}

In order to confirm differential expression of the genes selected by microarray analysis, RT-qPCR was used to validate these genes in an independent cohort. This cohort consisted of patients with RSV mono-infections to focus on the RSV specificity of the discovered genes. The final cohort consisted of 42 RSV-infected infants, of whom the patient characteristics are displayed in table 3. No significant differences were found between the groups, except for the parameters on which the clinical categorisation was based, age and RSV serotype.

To assess the overall sample quality, the expression of GAPDH in all samples was plotted (fig. 1). Generally, the amounts of RNA in the NPA samples were low; in a few samples, the amounts were too low to be able to measure gene expression, so these samples were excluded from further analysis.

These results clearly show a low but consistent expression in all samples, irrespective of the patient's health status. RT-qPCR analysis of our selected genes using nasopharyngeal samples from the validation cohort confirmed that the expression of all selected genes was significantly different, when comparing the recovery with acute samples. In this analysis, the acute group consisted of pooled expression data from all three groups: no supportive care, supplemental oxygen and mechanical ventilation. In case of TSPAN8, MUC13,

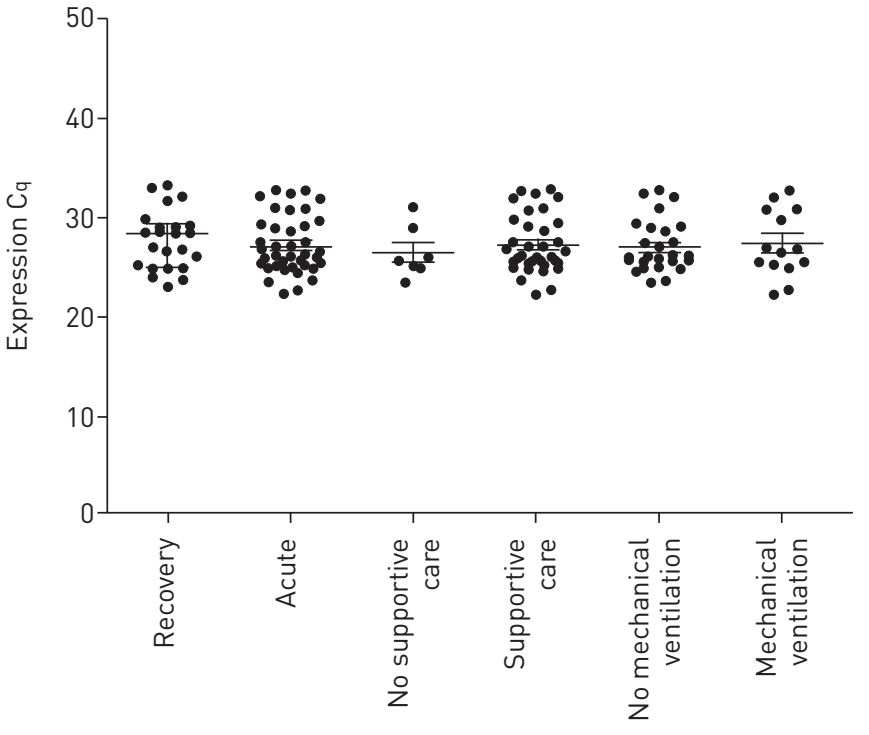

FIGURE 1 Nasopharyngeal expression of glyceraldehyde 3-phosphate dehydrogenase. Data are presented as median and interquartile range. Data were MannWhitney U-tested, two-sided (recovery, $n=23$; acute, $n=42$ ). No significant differences were found. $\mathrm{C}_{\mathrm{q}}$ : quantification cycle. 

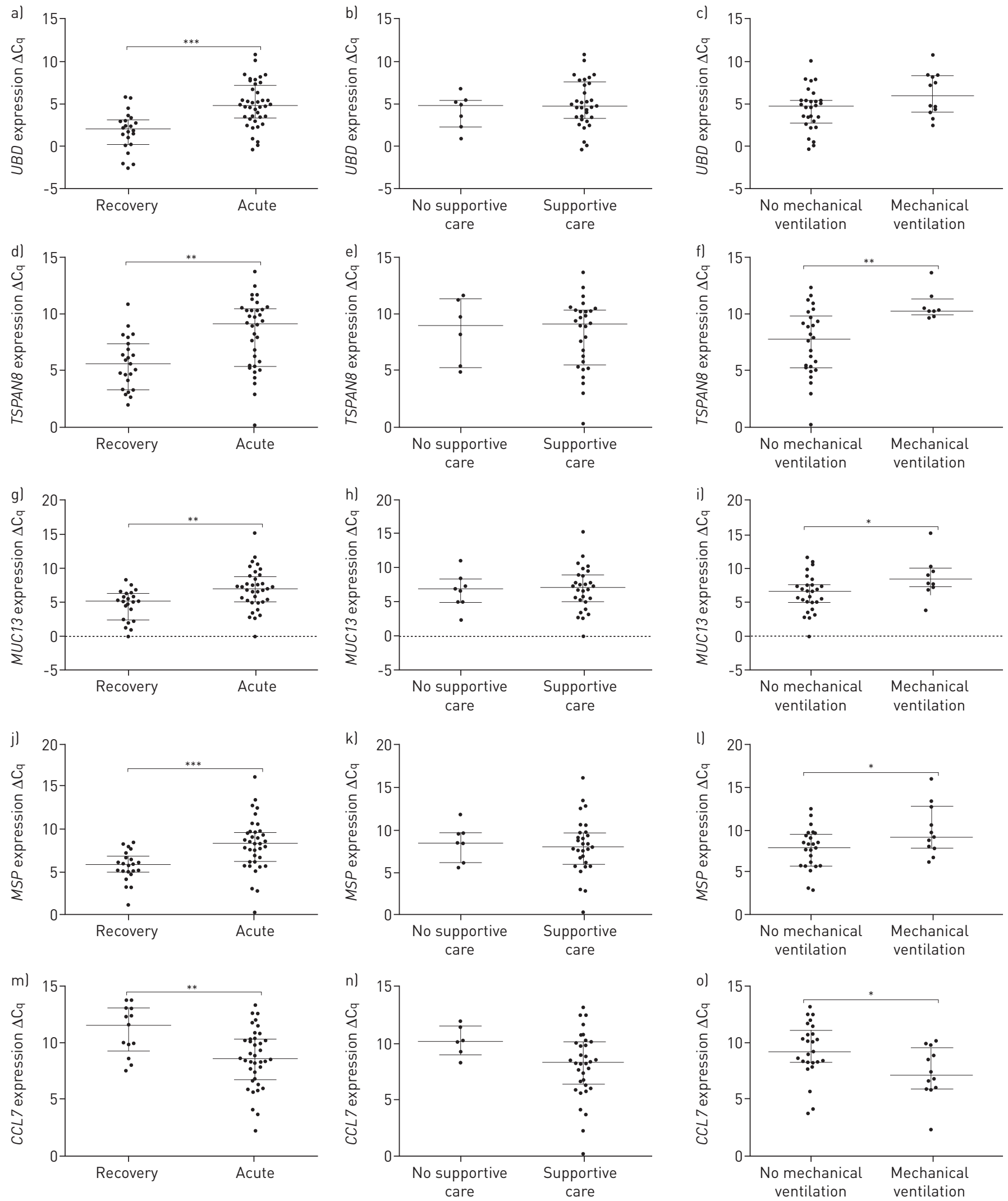

FIGURE 2 Nasopharyngeal expression of a-c) ubiquitin D (UBD), d-f) tetraspanin 8 (TSPAN8), g-i) mucin 13 (MUC13), j-1) $\beta$-microseminoprotein (MSP) and $\mathrm{m}-\mathrm{o}$ ) chemokine ligand 7 (CCL7) in recovery versus acute $(\mathrm{a}, \mathrm{d}, \mathrm{g}, \mathrm{j}$ and $\mathrm{m})$, need for supportive care $(\mathrm{b}, \mathrm{e}, \mathrm{h}, \mathrm{k}$ and $\mathrm{n}$ ) and need for mechanical ventilation ( $\mathrm{c}, \mathrm{f}$, $\mathrm{i}, 1$ and o). Data are presented as median and interquartile range. Data were Mann-Whitney U-tested, two-sided (recovery, $\mathrm{n}=23$; acute, $\mathrm{n}=42)$. $\Delta \mathrm{C}_{\mathrm{q}}$ : glyceraldehyde 3-phosphate dehydrogenase-normalised quantification cycle. ${ }^{*}: \mathrm{p}<0.05 ;{ }^{* *}: \mathrm{p}<0.01 ;{ }^{* *}: \mathrm{p}<0.001$. 
MSP and CCL7, the expression differed significantly in comparison 2 (the need for mechanical ventilation) (fig. 2). Subsequently, the correlation between the level of gene expression and known risk factors was assessed. Smoking at home, smoking during pregnancy, eczema, asthma and atopic family history did not show any correlation. For UBD only, a correlation with gestational age was found; however, gestational age is a well-known confounder, which does not exclude the value of $U B D$ as severity marker [23].

\section{Discussion}

This study shows that nasopharyngeal aspirates taken to diagnose the viral cause of infection can also be used to identify biomarkers by microarray analysis that are associated with severe RSV infections. The value of these markers was confirmed in an independent patient cohort. To our knowledge, this is the first study in which nasopharyngeal host gene expression was studied in the context of infection.

Gene expression analysis was unforeseen at the time of collection and a large part of the material was used for other studies; therefore, the quantity of the total material and the quality of the RNA isolated from the NPAs was suboptimal. The results of the GAPDH expression assay show that the absolute $\mathrm{C}_{\mathrm{q}}$ values are high, but consistent.

The microarray analysis resulted in the discovery of five significantly differentially expressed genes in the nasopharynx of infants with different degrees of severities of RSV infection. Remarkably, most genes are down-regulated in severely ill patients; this was confirmed with RT-qPCR. In addition, previously published microarray studies performed on gene expression profiles in peripheral blood of infants also showed a significant downregulation of genes $[16,17]$.

The method, as described in this study, with the aim to identify genes with potential prognostic value, has proven successful by the validation of TSPAN8, MUC13, MSP and CCL7. Although UBD did not discriminate between any acute group, it differed significantly between the recovered and acutely ill patients. This clearly underlines the value of our approach, in which microarray analysis was used to select differentially expressed genes in a discovery cohort, which were independently validated by qPCR and RT-qPCR in a separate cohort.

Besides CCL7, none of the genes identified in this study has previously been described to be associated with RSV. UBD participates in the ubiquitination process, labelling proteins for their degradation via the proteasome. MIELECH et al. [24] have shown that coronaviruses actively reduce ubiquitination of host cell proteins and observed their potential to modify the innate immune response. With regard to TSPAN8, YUE et al. [25] described its ability to override the adhesive features of CD151. On this note, CD151 is involved with the infectious entry of human papillomavirus [26], suggesting a significant infectious advantage for RSV when TSPAN8 is downregulated. Reduced amounts of MUC13 lead to decreased chemokine secretion in response to tumour necrosis factor- $\alpha$. This pro-inflammatory activity of MUC13 suggests that disrupted or inappropriate expression of MUC13 could predispose to infectious disease [27]. The downregulation of MSP was previously described in the context of rhinovirus infection [28]. MYGaTT et al. [29] reported virus-mediated silencing of $M S P$, which seems in accordance with our findings. Finally, significant upregulation of CCL7 has been observed in mice infected with rhinovirus [20] and in children during infection by different respiratory viruses, including RSV [30]. In addition, CCL7 was shown to be specifically upregulated when no mechanical ventilation was applied during RSV infection in mice and not differentially expressed when uninfected mice received mechanical ventilation [31], strongly suggesting that CCL7 upregulation is caused by RSV infection and is not a consequence of mechanical ventilation.

In conclusion, based on differential expression of TSPAN8, MUC13, MSP and CCL7 in readily available NPA samples, we can discriminate between severity of disease in RSV infected infants. This proves the potential of additional host gene expression analyses using regularly taken NPA samples, which, when combined with pathogen detection, would improve diagnosis of respiratory tract infections. Therefore, our findings demand further studies that might lead to the implementation of nasopharyngeal gene expression in diagnostics to guide clinical decisions in the context of respiratory tract infections.

\section{References}

1 Berger TM, Aebi C, Duppenthaler A, et al. Prospective population-based study of RSV-related intermediate care and intensive care unit admissions in Switzerland over a 4-year period (2001-2005). Infection 2009; 37: 109-116.

2 Purcell K, Fergie J. Driscoll Children's Hospital respiratory syncytial virus database: risk factors, treatment and hospital course in 3308 infants and young children, 1991 to 2002. Pediatr Infect Dis J 2004; 23: 418-423.

3 Mansbach JM, Clark S, Christopher NC, et al. Prospective multicenter study of bronchiolitis: predicting safe discharges from the emergency department. Pediatrics 2008; 121: 680-688.

4 Marlais M, Evans J, Abrahamson E. Clinical predictors of admission in infants with acute bronchiolitis. Arch Dis Child 2011; 96: 648-652. 
5 Damore D, Mansbach JM, Clark S, et al. Prospective multicenter bronchiolitis study: predicting intensive care unit admissions. Acad Emerg Med 2008; 15: 887-894.

6 Houben ML, Bont L, Wilbrink B, et al. Clinical prediction rule for RSV bronchiolitis in healthy newborns: prognostic birth cohort study. Pediatrics 2011; 127: 35-41.

7 Kneyber MC, Moons KG, de Groot R, et al. Prediction of duration of hospitalization in respiratory syncytial virus infection. Pediatr Pulmonol 2002; 33: 453-457.

8 Moler FW, Ohmit SE. Severity of illness models for respiratory syncytial virus-associated hospitalization. Am J Respir Crit Care Med 1999; 159: 1234-1240.

9 Brown L, Reiley DG, Jeng A, et al. Bronchiolitis: Can objective criteria predict eligibility for brief hospitalization? CJEM 2003; 5: 239-244.

10 Rietveld E, Vergouwe Y, Steyerberg EW, et al. Hospitalization for respiratory syncytial virus infection in young children: development of a clinical prediction rule. Pediatr Infect Dis J 2006; 25: 201-207.

11 Vieira RA, Diniz EM, Ceccon ME. Correlation between inflammatory mediators in the nasopharyngeal secretion and in the serum of children with lower respiratory tract infection caused by respiratory syncytial virus and disease severity. J Bras Pneumol 2010; 36: 59-66.

12 Marguet C, Bocquel N, Benichou J, et al. Neutrophil but not eosinophil inflammation is related to the severity of a first acute epidemic bronchiolitis in young infants. Pediatr Allergy Immunol 2008; 19: 157-165.

13 Bont L, Heijnen CJ, Kavelaars A, et al. Peripheral blood cytokine responses and disease severity in respiratory syncytial virus bronchiolitis. Eur Respir J 1999; 14: 144-149.

14 Mansbach JM, Piedra PA, Laham FR, et al. Nasopharyngeal lactate dehydrogenase concentrations predict bronchiolitis severity in a prospective multicenter emergency department study. Pediatr Infect Dis J 2012; 31: 767-769.

15 Brand HK, Ferwerda G, Preijers F, et al. CD4 ${ }^{+}$T-cell counts, interleukin-8 and CCL-5 plasma concentrations discriminate disease severity in children with RSV-infection. Pediatr Res 2013; 73: 187-793.

16 Ramilo O, Allman W, Chung W, et al. Gene expression patterns in blood leukocytes discriminate patients with acute infections. Blood 2007; 109: 2066-2077.

17 Mejias A, Dimo B, Suarez NM, et al. Whole blood gene expression profiles to assess pathogenesis and disease severity in infants with respiratory syncytial virus infection. PLoS Med 2013; 10: e1001549.

18 Brand KH, Ahout IM, de Groot R, et al. Use of MMP-8 and MMP-9 to assess disease severity in children with viral lower respiratory tract infections. J Med Virol 2012; 84: 1471-1480.

19 Templeton KE, Scheltinga SA, Beersma MF, et al. Rapid and sensitive method using multiplex real-time PCR for diagnosis of infections by influenza a and influenza B viruses, respiratory syncytial virus, and parainfluenza viruses 1,2, 3, and 4. J Clin Microbiol 2004; 42: 1564-1569.

20 Schneider D, Hong JY, Bowman ER, et al. Macrophage/epithelial cell CCL2 contributes to rhinovirus-induced hyperresponsiveness and inflammation in a mouse model of allergic airways disease. Am J Physiol Lung Cell Mol Physiol 2013; 304: L162-L169.

21 Staal FJ, van der Burg M, Wessels LF, et al. DNA microarrays for comparison of gene expression profiles between diagnosis and relapse in precursor-B acute lymphoblastic leukemia: choice of technique and purification influence the identification of potential diagnostic markers. Leukemia 2003; 17: 1324-1332.

22 Klipper-Aurbach Y, Wasserman M, Braunspiegel-Weintrob N, et al. Mathematical formulae for the prediction of the residual beta cell function during the first two years of disease in children and adolescents with insulin-dependent diabetes mellitus. Med Hypotheses 1995; 45: 486-490.

23 Clark RH, Kelleher AS, Chace DH, et al. Gestational age and age at sampling influence metabolic profiles in premature infants. Pediatrics 2014; 134: e37-e46.

24 Mielech AM, Kilianski A, Baez-Santos YM, et al. MERS-CoV papain-like protease has deISGylating and deubiquitinating activities. Virology 2014; 450: 64-70.

25 Yue SJ, Mu W, Zoller M. Tspan8 and CD151 promote metastasis by distinct mechanisms. Eur J Cancer 2013; 49: 2934-2948.

26 Scheffer KD, Gawlitza A, Spoden GA, et al. Tetraspanin CD151 mediates papillomavirus type 16 endocytosis. J Virol 2013; 87: 3435-3446.

27 Sheng YH, Triyana S, Wang R, et al. MUC1 and MUC13 differentially regulate epithelial inflammation in response to inflammatory and infectious stimuli. Mucosal Immunol 2013; 6: 557-568.

28 Bochkov YA, Hanson KM, Keles S, et al. Rhinovirus-induced modulation of gene expression in bronchial epithelial cells from subjects with asthma. Mucosal Immunol 2010; 3: 69-80.

29 Mygatt JG, Singhal A, Sukumar G, et al. Oncogenic herpesvirus HHV-8 promotes androgen-independent prostate cancer growth. Cancer Res 2013; 73: 5695-5708.

30 Lewis TC, Henderson TA, Carpenter AR, et al. Nasal cytokine responses to natural colds in asthmatic children. Clin Exp Allergy 2012; 42: 1734-1744.

31 Hennus MP, Janssen R, Pennings JLA, et al. Host response to mechanical ventilation for viral respiratory tract infection. Eur Respir J 2012; 40: 1508-1515. 